\title{
On five new species of the millipede family Paradoxosomatidae from Papua New Guinea (Diplopoda: Polydesmida)
}

\section{O пяти новых видах диплопод семейства Paradoxosomatidae из Папуа - Новой Гвинеи (Diplopoda: Polydesmida)}

\author{
Sergei I. Golovatch ${ }^{1}$, Pavel Stoev ${ }^{2}$ \\ Сергей И. Головач ${ }^{1}$, Павел Стоев²
}

\footnotetext{
${ }^{1}$ Institute for Problems of Ecology and Evolution, Russian Academy of Sciences, Leninsky prospekt 33, Moscow 119071 Russia.

${ }^{1}$ Институт проблем экологии и эволюции РАН, Ленинский проспект, 33, Москва 119071 Россия.

${ }^{2}$ National Museum of Natural History, Bulgarian Academy of Sciences, Boulevard Tsar Osvoboditel 1, Sofia 1000 Bulgaria.

${ }^{2}$ Национален природонаучен музей БАН, бул. Цар Освободител 1, София 1000 България.
}

KEY WORDS: Millipedes, Caloma, Nothrosoma, Dendrogonopus, taxonomy, new species, key, New Guinea.

КЛЮЧЕВЫЕ СЛОВА: Диплоподы, Caloma, Nothrosoma, Dendrogonopus, таксономия, новые виды, ключ, Новая Гвинея.

ABSTRACT. Reviews of three Papuan genera are presented: Caloma Chamberlin, 1945 (Tectoporini), Nothrosoma Attems, 1929 (Eustrongylosomatini) and Dendrogonopus Jeekel, 1964 (Aschistodesmini). Five new species are described from Papua New Guinea: Caloma pallidum sp.n., Nothrosoma crassipes sp.n., N. mediapes sp.n., N. longipes sp.n. and Dendrogonopus beroni sp.n. Keys to all three, six and three species of these genera are given, respectively, with all distributions mapped.

РЕЗЮМЕ. Представлены обзоры трех папуасских родов: Caloma Chamberlin, 1945 (Tectoporini), Nothrosoma Attems, 1929 (Eustrongylosomatini) и Dendrogonopus Jeekel, 1964 (Aschistodesmini). Описаны пять новых видов из Папуа - Новой Гвинеи: Caloma pallidum sp.n., Nothrosoma crassipes sp.n., $N$. mediapes sp.n., $N$. longipes sp.n. и Dendrogonopus beroni sp.n. Составлены ключи соответственно для всех трех, шести и трех видов этих родов, а также дана карта их распространения.

\section{Introduction}

This paper concludes our joint work on the paradoxosomatid millipedes collected in 1975 in Papua New Guinea by the British Speleological Expedition (B.S.E.), mainly by Petar Beron. This collection is housed in the National Museum of Natural History (NMNHS), Sofia, Bulgaria. Previous information on Paradoxosomatidae from that collection can be found in Hoffman [1978, 2005] and Golovatch \& Stoev [2009, 2010, 2011, 2014].

The present contribution deals with three Papuan genera: Nothrosoma Attems, 1929, Caloma Chamber- lin, 1945, and Dendrogonopus Jeekel, 1964. Although they belong to different tribes, it seems more reasonable to publish the remaining five new species from that great collection together. Furthermore, reviews of and keys to all currently known species of these three genera are given, with their distributions mapped (Fig. 1).

\section{Taxonomic part}

Tribe Eustrongylosomatini Jeekel, 1968

Genus Nothrosoma Attems, 1929

Type species: Orthomorpha acuta Attems, 1914, by original designation.

SPECIES INCLUDED: Nothrosoma acutum (Attems, 1914), from Mosso, Papua Province, Indonesia [Attems, 1914]; N. loriae (Silvestri, 1895), from Hughibagu, Central Province, Papua New Guinea (an uncertain locality, possibly close to Moroka) and Moroka, Central Province, Bartholomew Mountains, Papua New Guinea [Silvestri, 1895] (= Meroka, according to Holthuis [1974], a locality near Mount Wori-Wori (= Vori Vori Bluff), $09^{\circ} 25^{\prime} \mathrm{S}, 147^{\circ} 41^{\prime} \mathrm{E}$ [Helgen et al., 2008]); $N$. beroni Hoffman, 1978, from a cave in Finim Tel Plateau, Papua New Guinea [Hoffman 1978]; N. crassipes sp.n., from Telefomin area, Papua New Guinea; $N$. mediapes sp.n., from near the village of Bultem, Papua New Guinea, and N. longipes sp.n., from near Tifalmin, Papua New Guinea.

REMARKS. The small tribe Eustrongylosomatini contains only five genera, chiefly from the Papuan region, with only a single genus and species occurring as far away as the Seychelles [Golovatch \& Stoev, 2011]. It is defined through the gonopod showing an 


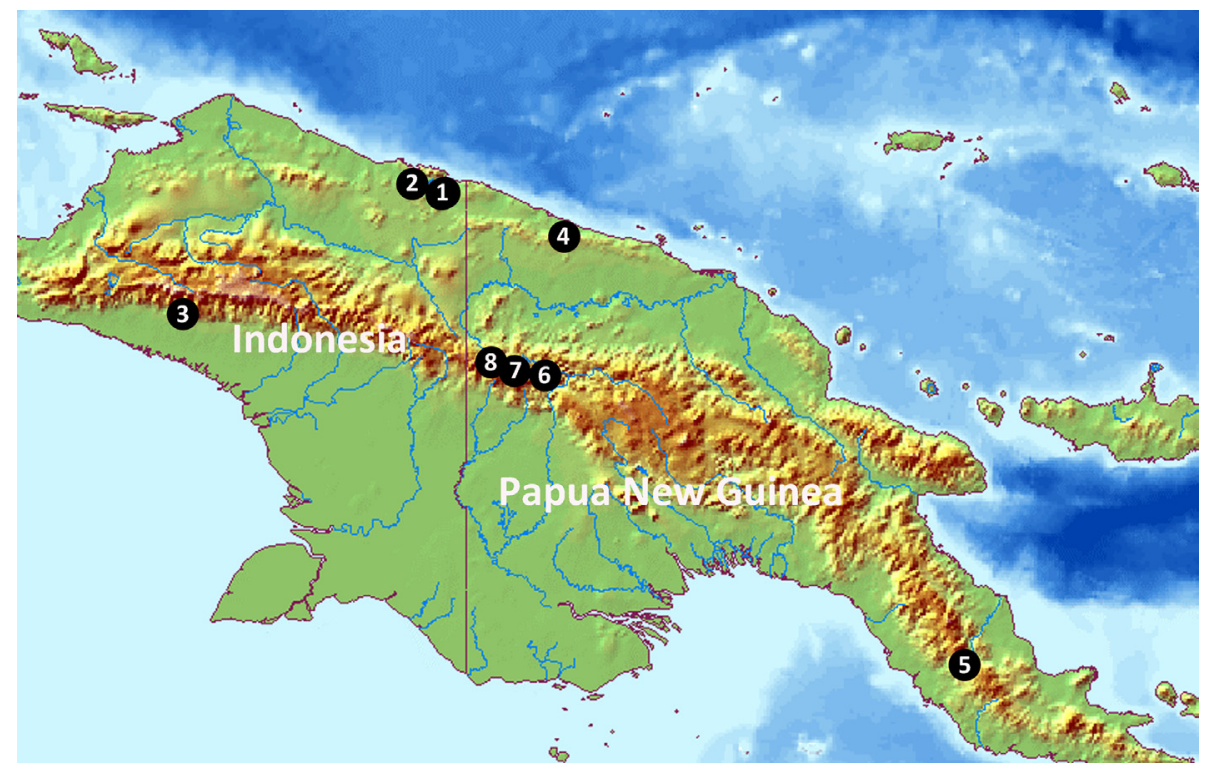

Fig. 1. Distribution of the millipede genera Nothrosoma, Caloma and Dendrogonopus in New Guinea: $1-N$. acutum, $2-C$. agametum, $3-D$. robustus, $4-C$. insulare, $5-N$. loriae, D. maculatus, $6-$ D. beroni sp.n., C. pallidum sp.n., N. crassipes sp.n., 7 - C. agametum, $N$. beroni, $N$. longipes sp.n., $8-N$. mediapes sp.n.

Рис. 1. Распространение диплопод родов Nothrosoma, Caloma и Dendrogonopus в Новой Гвинее: 1 - N. acutum, 2 - C. agametum, $3-$ D. robustus, $4-$ C. insulare, $5-N$. loriae, D. maculatus, $6-D$. beroni sp.n., C. pallidum sp.n., N. crassipes sp.n., 7 - C. agametum, $N$. beroni, N. longipes sp.n., $8-N$. mediapes sp.n.

elongated and modestly twisted femorite typically supplied with one or two longitudinal lobes, supporting a seminal groove mesally between these lobes, and ending up by a distinct distofemoral sulcus basal to an apical outgrowth, often also with one or more nearby processes, and a subcircular, long and often rather complex solenophore with typical lamina lateralis and lamina medialis, both sheathing a long flagelliform solenomere. Among them, Nothrosoma is distinguished by the particularly prominent, wing-like paraterga. Only three species have hitherto been described, all keyed [Hoffman, 1978].

Generally speaking, the degree of development of paraterga alone is a poor character to define genera in Paradoxosomatidae [Jeekel, 1968]. Therefore, the generic status of Nothrosoma is still to be questioned, especially versus the most species-rich genus Eustrongylosoma Silvestri, 1896, as their basic gonopod traits are the same. However, a detailed analysis of the relationships between the genera of Eustrongylosomatini lies beyond the scope of the present note.

Below are descriptions of another three new Nothrosoma species.

\section{Nothrosoma crassipes sp.n.}

Figs 2-10.

HOLOTYPE $\sigma^{7}$ (broken into several pieces) (NMNHS), Papua New Guinea: Upper Sepik Province, Telefomin area, September 1975, British Speleological Expedition (leg. P. Beron \& Ph. Chapman).

NAME. To emphasize the relatively robust legs, adjective.
DIAGNOSIS. Differs from congeners by less prominent paraterga (Figs $2 \& 3$ ), the very poorly concave tip of the epiproct (Fig. 4), the linguiform sternal lobe between $\sigma^{T}$ coxae 4 (Fig. 6), and the ribbon-shaped and trifid tip of the gonopods showing a rounded base of lobe $\boldsymbol{m}$ (Fig. 8) (see also Key below).

DESCRIPTION. Length ca $20 \mathrm{~mm}$, width of midbody pro- and metazonae 2.0 and $3.0 \mathrm{~mm}$, respectively. Coloration rather uniformly brown; antennae, head and first three segments a little darker brown; paraterga, venter and legs light yellowish brown to light greybrown.

In width, segment $2=3<$ head $=$ collum $<5<6-$ 16; body gently tapering towards telson thereafter. Clypeolabral region densely setose, vertigial region bare. Antennae long and slender, antennomere $2=3>$ subequal 4-6, reaching behind segment 3 when stretched dorsally. Collum very broadly and regularly rounded on sides, clearly rimmed. Tegument smooth and shiny, metaterga at most faintly rugulose, only surface below paraterga microgranulate. Paraterga 2 with a small, but evident, lateral tooth at front corner; caudal corner acutangular (ca $75^{\circ}$ ), nearly pointed, slightly produced behind rear tergal margin. All following paraterga subhorizontal, set rather high (at about $1 / 3^{\text {rd }}$ of midbody height), increasingly well drawn behind rear tergal margin, devoid of lateral indentations, slightly thicker in pore-bearing segments, only in $19^{\text {th }}$ rather small and low teeth (Figs $2 \& 3$ ). Paraterga delimited by a sulcus only dorsally; callus rather indistinct and medium-sized even in pore-bearing segments, considerably narrower in poreless ones. Tergal setae abraded, setation pattern 


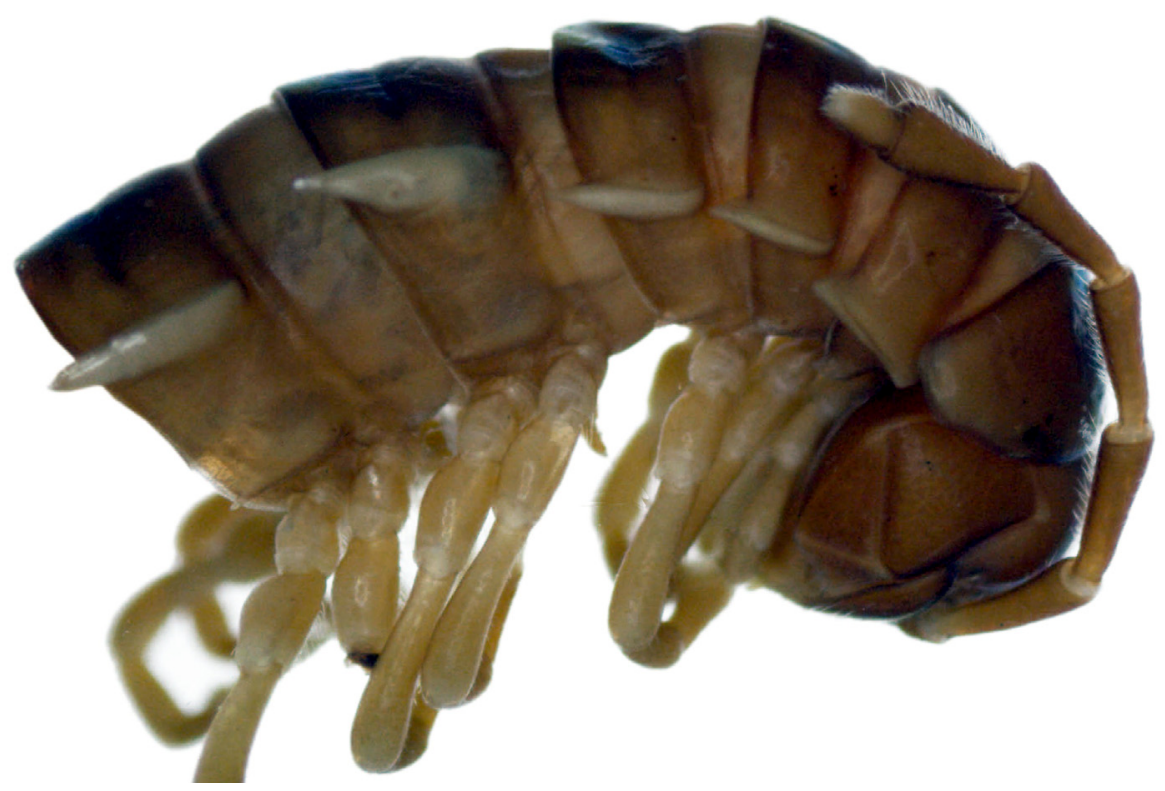

Fig. 2. Nothrosoma crassipes sp.n., holotype, head and anterior body segments, lateral view. Photo taken not to scale.

Pис. 2. Nothrosoma crassipes sp.n., голотип, голова и передние сегменты тела, сбоку. Фотография сделана без масштаба.

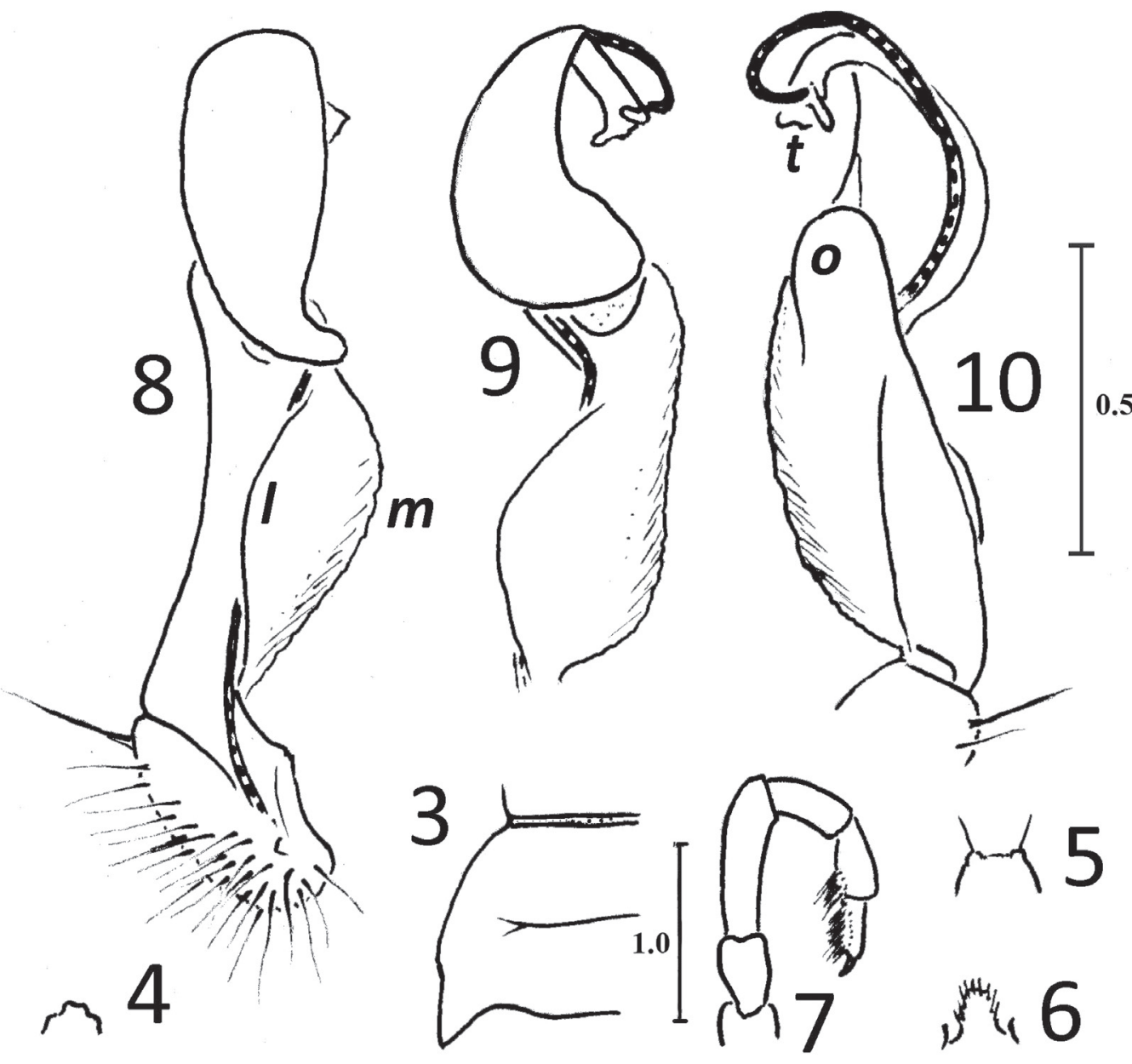

Figs 3-10. Nothrosoma crassipes sp.n., holotype: 3 - midbody segment, dorsal view; 4 - tip of epiproct, dorsal view; 5 hypoproct, ventral view; 6 - sternal lobe between coxae 4 , subcaudal view; 7 - leg 10, lateral view; 8-10 — right gonopod, mesal, dorsal and lateral views, respectively. Scale bars: $1.0(3-7)$ and $0.5 \mathrm{~mm}(8-10)$.

Рис. 3-10. Nothrosoma crassipes sp.n., голотип: 3 - среднетуловищный сегмент, сверху; 4 - вершина эпипрокта, сверху; 5 гипопрокт, снизу; 6 - стернальная пластина между тазиками 4, почти сзади; 7 - нога 10, сбоку; 8-10 - правый гонопод, соответственно изнутри, сверху и сбоку. Масштаб: 1,0 (3-7) и 0,5 мм (8-10). 


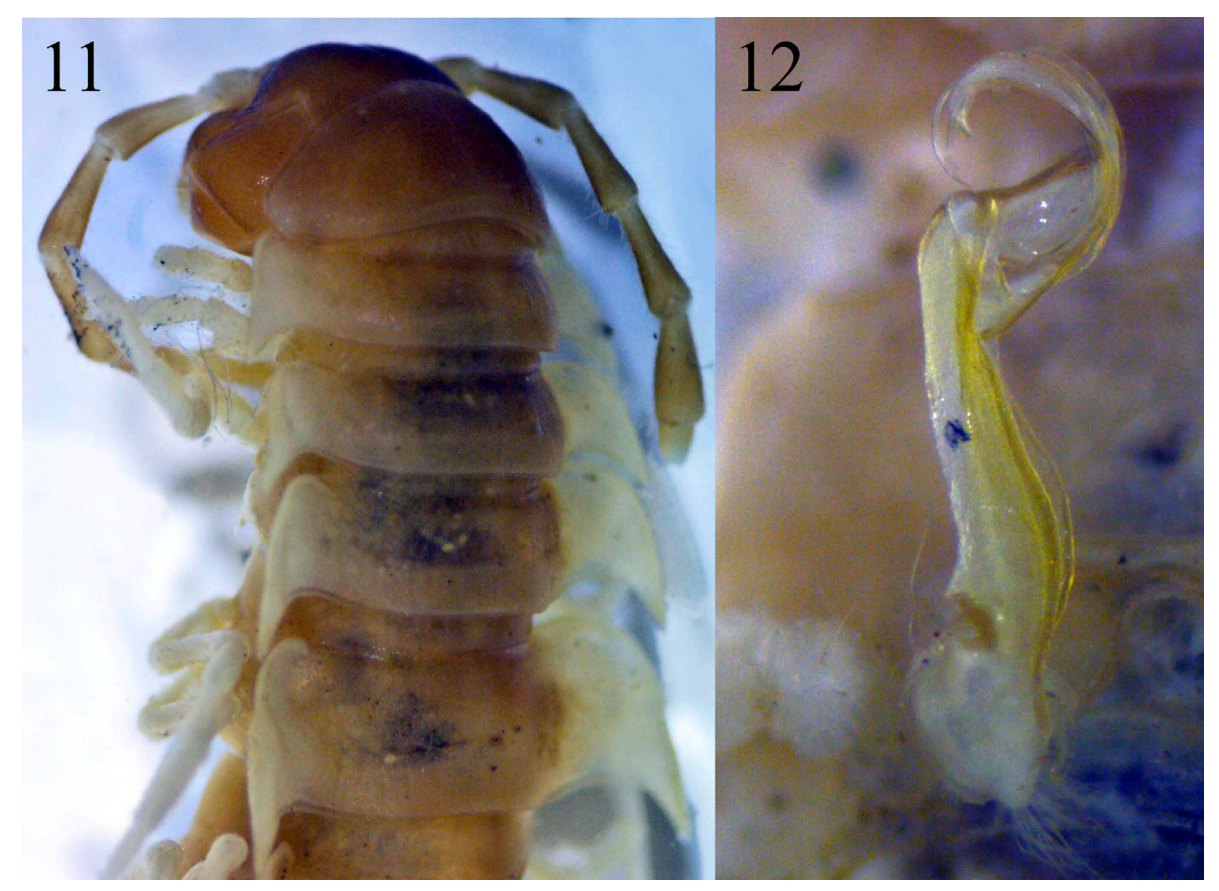

Figs 11 \& 12. Nothrosoma mediapes sp.n., holotype: 11 — head and anterior body segments, dorsolateral view; 12 — right gonopod in situ, ventral view. Photos taken not to scale.

Рис. 11 и 12. Nothrosoma mediapes sp.n., голотип: 11 - голова и передние сегменты тела, одновременно сверху и сбоку; 12 правый гонопод in situ, снизу. Фотографии сделаны без масштаба.

untraceable, probably $1+1$ or $2+2$ setae in a front row only. Ozopores ventrolateral, lying at ca $1 / 3^{\text {rd }}$ length of paraterga in front of caudal margin. Axial line nearly wanting, only in places very poorly traceable on metaterga. Transverse metatergal sulci incomplete and especially thin in segment 4 , complete, rather deep and almost reaching bases of paratega in segments 5-18, absent from $19^{\text {th }}$. Stricture between pro- and metazonae evident, but at most very faintly striolate down to below paraterga. Pleurosternal carinae small, but evident caudal spines increasingly strongly reduced until segment 8 , remaining only small knobs on $9^{\text {th }}$ and $10^{\text {th }}$, missing thereafter $\left(\sigma^{7}\right)$. Epiproct (Fig. 4) very short, faintly concave at tip; subapical lateral papillae very evident. Hypoproct (Fig. 5) subtrapeziform, caudolateral setae borne on quite evident papillae. Sterna densely setose; cross-impressions faint, axial impressions especially so; lobe between coxae 4 rather high, densely setose, linguiform (Fig. 6). Legs only relatively short, clearly crassate $\left(O^{7}\right)$, ca $1.7-1.8$ times as long as midbody height (Fig. 7); distotibial and tarsal brushes present until legs of segment 8 , gradually thinning out thereafter.

Gonopods (Figs 8-10) with rather short, distoventrally sparsely setose coxites, prefemoral part even shorter, about $1 / 3^{\text {rd }}$ as long as acropodite; femorite with two distinct lobes, one mesal $(l)$, the other dorsal, slightly serrate $(\boldsymbol{m})$. Solenophore rather simple, about as long as a flagelliform solenomere; distolateral outgrowth of femorite $(\boldsymbol{o})$ modest; tip $(\boldsymbol{t})$ of solenophore narrow and trifid.

\section{Nothrosoma mediapes sp.n.}

Figs 11-19.

HOLOTYPE $\sigma^{7}$ (NMNHS), Papua New Guinea: Western Province, Bultem Village, 8 October 1975, British Speleological Expedition (leg. P. Beron).

NAME. To emphasize the medium-sized legs, adjective.

DIAGNOSIS. Differs from congeners by having more prominent paraterga (Figs $11 \& 13$ ), the epiproct tip being divided into two spines (Fig. 14), by the roundly subtriangular sternal lobe between $O^{7}$ coxae 4 (Fig. 16), and a subacuminate and bifid tip of the gonopods showing a subquadrate base of lobe $\boldsymbol{m}$ (Fig. 19) (see also Key below).

DESCRIPTION. Length ca $23 \mathrm{~mm}$, width of midbody pro- and metazonae 2.5 and $4.0 \mathrm{~mm}$, respectively. Coloration uniformly light yellowish to pallid, only antennae, head and 3-4 following segments very light brown.

All characters as in $N$. crassipes, except as follows:

In width, head $<$ collum $<$ segment $3=4<2=5<$ 6-16; body gently tapering towards telson thereafter. Clypeolabral region densely setose, vertigial region with a few setae only. Collum less broadly, but regularly rounded on sides, poorly rimmed. Surface below paraterga faintly microgranulate. Paraterga 2 with caudal corner sharp $\left(\mathrm{ca} 50^{\circ}\right)$ and pointed, like all following paraterga very clearly drawn behind rear tergal margin, broader than in $N$. crassipes sp.n. Paraterga very thin, poriferous paraterga with evident shoulders, in posteri- 


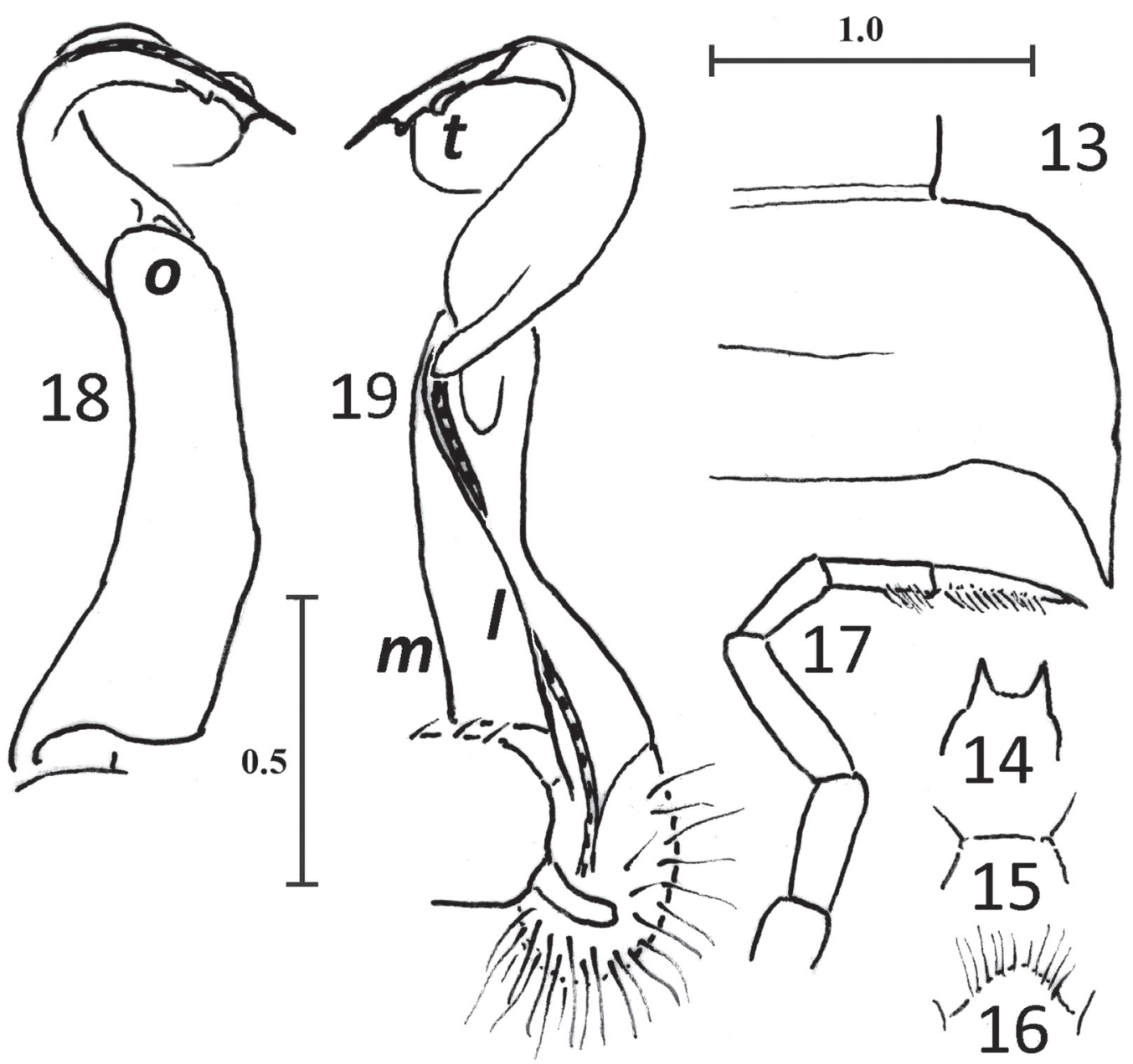

Figs 13-19. Nothrosoma mediapes sp.n., holotype: 13 - midbody segment, dorsal view; 14 - tip of epiproct, dorsal view; 15 hypoproct, ventral view; 16 - sternal lobe between coxae 4, subcaudal view; 17 - leg 9, lateral view; 18 \& 19 - left gonopod, lateral and mesal views, respectively. Scale bars: $1.0(13-17)$ and $0.5 \mathrm{~mm}(18 \& 19)$.

Рис. 13-19. Nothrosoma mediapes sp.n., голотип: 13 - среднетуловищный сегмент, сверху; 14 - вершина эпипрокта, сверху; 15 - гипопрокт, снизу; 16 - стернальная пластина между тазиками 4, почти сзади; 17 - нога 9, сбоку; 18 и 19 - левый гонопод, соответственно сбоку и изнутри. Масштаб: 1,0 (13-17) и 0,5 мм (18 и 19).

or $1 / 3^{\text {rd }}$ of body caudal parts of paraterga $>1 / 3^{\text {rd }}$ length of paraterga. Calluses very thin and poorly delimited, shoulders best so. Tergal setae short, only about $1 / 5^{\text {th }}$ of metatergal length, pattern at least $1+1(2+2$ ? $)$ setae in an anterior row. Ozopores ventrolateral, lying at about midway of paraterga. Transverse metatergal sulci very shallow lines far from reaching bases of paraterga, visible only in segments $5-18$. Stricture between pro- and metazonae rather shallow and smooth. Pleurosternal carinae small, sharp caudal teeth gradually reduced until segment 18. Epiproct (Fig. 14) short, with two strong spines at tip, subapical lateral papillae very small. Hypoproct (Fig. 15) subtrapeziform, caudolateral papillae very small, with long setae. Lobe between coxae 4 roundly subtriangular (Fig. 16). Legs (Fig. 17) ca 2.0 times as long as midbody height $\left(\sigma^{7}\right)$; distotibial and tarsal brushes traceable only until legs of segment 7, abruptly thinning out thereafter.

Gonopods (Figs 12, 18 \& 19) with rather short coxites, prefemoral part even shorter, about $1 / 4^{\text {th }}$ as long as acropodite; femorite long and slender, lobe $\boldsymbol{l}$ very low and inconspicuous, lobe $\boldsymbol{m}$ relatively small and subrectangular at base; distolateral outgrowth of femorite $(\boldsymbol{o})$ regularly rounded and inconspicuous. Solenophore subacuminate, a little shorter than solenomere, tip ( $\boldsymbol{t})$ bifid.

\section{Nothrosoma longipes sp.n.}

Figs 20-28.

HOLOTYPE $0^{7}$ (NMNHS), Papua New Guinea: West Sepik Province, Tifalmin, alt. 1700 m, 14 September 1975, British Speleological Expedition (leg. P. Beron).

NAME. To emphasize the especially long legs, adjective.

DIAGNOSIS. Differs from congeners by having spiniform paraterga (Figs 20, $21 \& 25$ ), especially long legs (ca 2.2 times as long as midbody height) (Fig. 25), and a ribbon-shaped and bifid apical part of the solenophore, as in Figs $27 \& 28$ (see also Key below). 


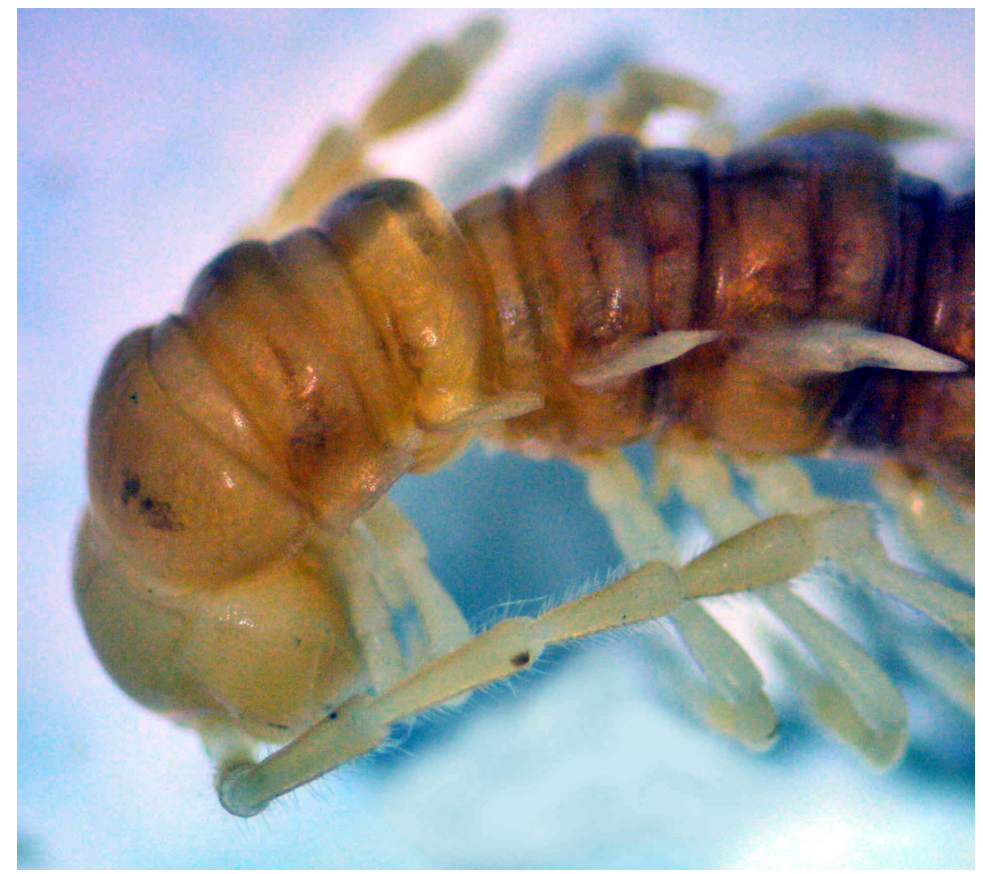

Fig. 20. Nothrosoma longipes sp.n., holotype, head and anterior body segments, dorsolateral view. Photo taken not to scale.

Рис. 20. Nothrosoma longipes sp.n., голотип, голова и передние сегменты тела, одновременно сверху и сбоку. Фотография сделана без масштаба.

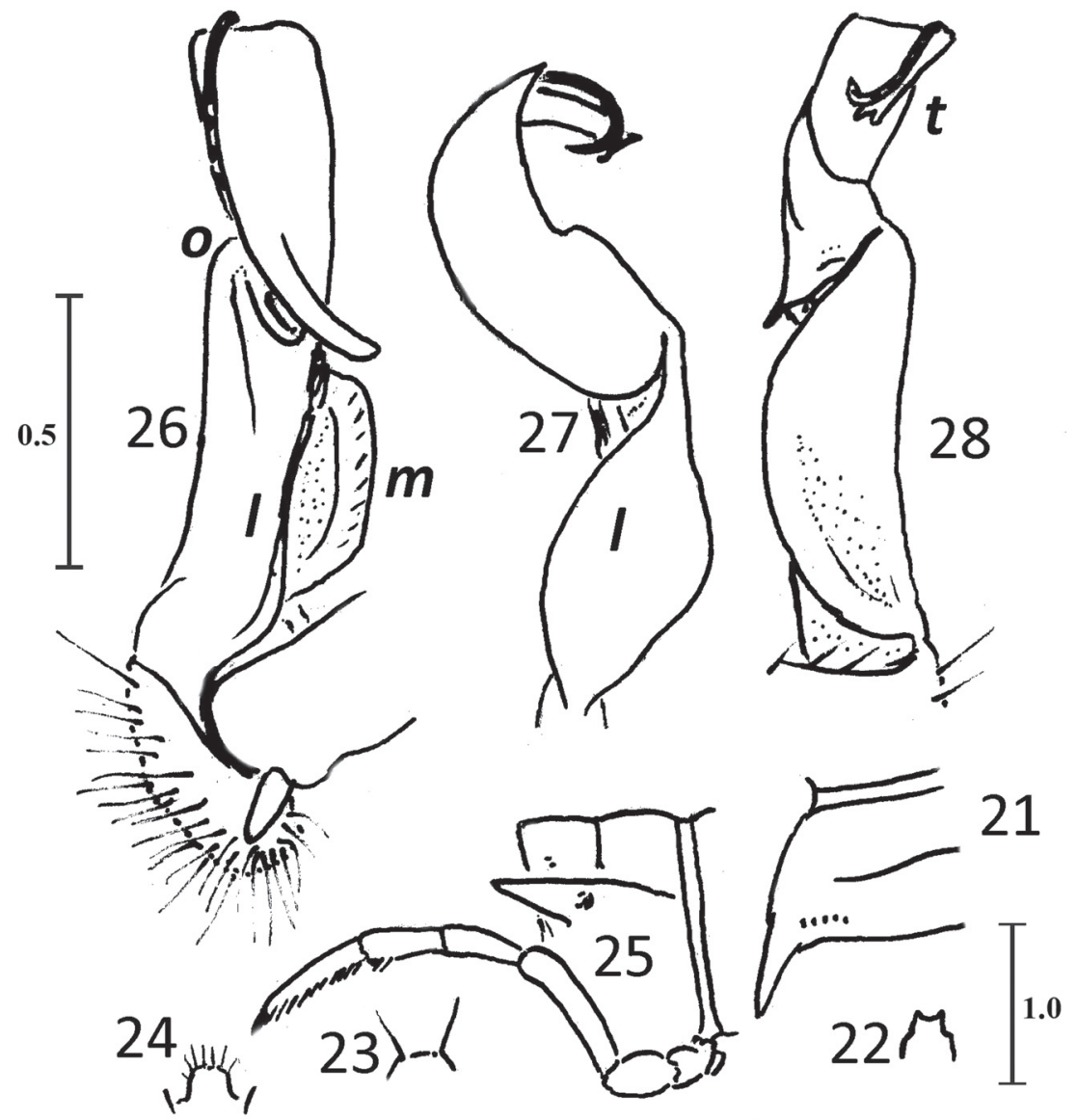

Figs 21-28. Nothrosoma longipes sp.n., holotype: $21 \& 25$ - midbody segment, dorsal and lateral views, respectively; 22 - tip of epiproct, dorsal view; 23 - hypoproct, ventral view; 24 - sternal lobe between coxae 4, subcaudal view; 26-28 - right gonopod, mesal, dorsal and lateral views, respectively. Scale bars: 1.0 (21-25) and $0.5 \mathrm{~mm}(26-28)$.

Рис. 21-28. Nothrosoma longipes sp.n., голотип: 21 и 25 - среднетуловищный сегмент, соответственно сверху и сбоку; 22 вершина эпипрокта, сверху; 23 - гипопрокт, снизу; 24 - стернальная пластина между тазиками 4, почти сзади; 26-28 - правый гонопод, соответственно изнутри, сверху и сбоку. Масштаб: 1,0 (21-25) и 0,5 мм (26-28). 
DESCRIPTION. Length ca $20 \mathrm{~mm}$, width of midbody pro- and metazonae 1.7 and $2.8 \mathrm{~mm}$, respectively. Coloration rather uniformly brown, but head, collum, antennae, legs, paraterga and telson light brown to whitish.

All characters as in $N$. crassipes, except as follows:

In width, collum $<$ head $=$ segment $3=4<2<5<$ 6-16; body gently tapering towards telson thereafter. Antennae long, slender, slightly clavate, antennomere $2=3>4>5>6$, reaching beyond middle of segment 4 when stretched dorsally. Collum very broadly and regularly rounded on sides, slightly rimmed. Paraterga 2 with caudal corner acutangular $\left(\mathrm{ca} \mathrm{50} 0^{\circ}\right)$, nearly pointed, clearly produced behind rear tergal margin. All following paraterga spiniform. Calluses and shoulders wanting. Tergal setae fully abraded, setation pattern untraceable. Ozopores ventrolateral, lying at about midway of paraterga. Axial line very poorly developed, but traceable on metaterga. Transverse sulci on metaterga deep, complete and reaching bases of paraterga in segments $5-18$, absent from $19^{\text {th }}$. Stricture between proand metazonae smooth, very finely striolate. Pleurosternal carinae small, caudal spines until segment 10, missing thereafter. Epiproct (Fig. 22) short, deeply emarginate at tip; subapical lateral papillae modest. Hypoproct (Fig. 23) subtrapeziform, caudolateral papillae very small, with long setae. Lobe between coxae 4 subquadrate, modest, densely setose (Fig. 24). Legs especially long (Fig. 25), ca 2.2 times as long as midbody height; distotibial and tarsal brushes present until legs of segment 11, gradually thinning out thereafter.

Gonopods (Figs 26-28) with rather short coxites, prefemoral part about $1 / 3^{\text {rd }}$ as long as acropodite; femorite with two distinct lobes, lobe $\boldsymbol{l}$ long, but low, lobe $\boldsymbol{m}$ prominent and rounded. Solenomere only very slightly longer than solenophore; distolateral outgrowth of femorite (o) low and rounded; tip $(\boldsymbol{t})$ of solenophore ribbon-shaped and bifid.

\section{Key to Nothrosoma SPECIES}

1.Paraterga spiniform, but lying below dorsum (Figs 20, 21, 25 ), legs very long, about 2.2 times as long as midbody height (Fig. 25); gonopods as in Figs 26-28

.N. longipes sp.n.

- Paraterga keel-shaped, sometimes some lying above dorsum; legs shorter; gonopods different

2.Poriferous paraterga each with a clear indentation; length to width ratio: $20 \times 2.6 \mathrm{~mm}$; light testaceous; tip of epiproct very short and subtruncate, subapical papillae prominent; paraterga keel-shaped, but close to spiniform, ozopores lying clearly ventrally on paraterga; male unknown, cave N. beroni

- All paraterga without indentations; light or dark; epiproct different; ozopores lying clearly laterally on paraterga; epigean .....

3. Larger, length to width ratio: $28 \times 3.8-4.1 \mathrm{~mm}$; black; epiproct deeply emarginate; paraterga upturned above dorsum; sternal lobe subquadrate to semi-circular; gonopods: lobe $\boldsymbol{l}$ low and short; lobe $\boldsymbol{m}$ low and rounded; sternal lobe a rounded lamella, tip of solenophore bifid, with a rounded lobe and a sharp spine .............. . loriae

- Smaller; usually lighter; epiproct usually not so deeply emarginate; paraterga lying below dorsum; sternal lobe usually different; gonopods different ........................... 4

4.Body nearly black; head almost without setae; sternal lobe between $O^{7}$ coxae 4 divided into two setose tubercles; epiproct truncate; gonopods: tip of solenophore ribbonshaped, broad and with several spines; lobe $\boldsymbol{m}$ very low N. acutum

- Body lighter, at least clypeolabral region densely setose; sternal lobe between $\sigma^{7}$ coxae 4 always single; epiproct and gonopods different ............................................ 5

5. Paraterga more prominent (Figs $11 \& 13$ ), tip of epiproct divided into two spines (Fig. 14); sternal lobe between $\sigma^{7}$ coxae 4 roundly subtriangular (Fig. 16); gonopods: tip subacuminate and bifid, base of lobe $\boldsymbol{m}$ subquadrate (Figs 11, $18 \&$ 19) ........................... N. mediapes sp.n.

- Paraterga less prominent (Figs $2 \& 3$ ), tip of epiproct very poorly concave (Fig. 4); sternal lobe between $\sigma^{7}$ coxae 4 linguiform (Fig. 6); gonopods: tip ribbon-shaped and trifid, base of lobe $\boldsymbol{m}$ rounded (Figs 8-10)

$$
\text { N. crassipes sp.n. }
$$

Tribe Tectoporini Jeekel, 1968

Genus Caloma Chamberlin, 1945

Type species: Caloma agametum Chamberlin, 1945, by original designation.

SPECIES INCLUDED: Caloma agametum Chamberlin, 1945, from 'Prauwenbivak' $\left(138.35^{\circ} \mathrm{E}, 3.15^{\circ} \mathrm{S}\right.$, ca 40 km SSW of Sukarnapura, Papua Province, Indonesia [Chamberlin, 1945; Hoffman, 2005], and Finim Tel Plateau, Papua New Guinea [Hoffman, 2005; Golovatch \& Stoev, 2009]; C. insulare (Silvestri, 1899), from 'Berlinhafen' (now Aitape, at $142.35^{\circ} \mathrm{E}, 3.13^{\circ} \mathrm{S}$, a settlement on the coast of North Sepik District, Papua New Guinea [Silvestri, 1899; Hoffman, 2005]; and $C$. pallidum sp.n., from Cave Bolam Tigiin Tem, Telefomin, Papua New Guinea.

\section{Caloma pallidum sp.n.}

Figs 29-34.

HOLOTYPE O $0^{T}$ (NMNHS), Papua New Guinea, West Sepik Province, Telefomin, Cave Bolam Tigiin Tem, alt. 2100 m, 25 August 1975, B.S.E. (leg. P. Beron).

PARATYPES: 1 freshly moulted $\sigma^{7}, 1$ ( 1 (NMNHS), same locality, date and collector as holotype.

NAME. To emphasize the pallid coloration, adjective.

DIAGNOSIS. Based on gonopod structure, this new species is especially similar to $C$. insulare, sharing a stout femorite and a slender and trifid solenophore. However, C. pallidum sp.n. differs in a more elaborate and slightly longer femoral part, a shorter solenomere and wider apical lobes (see also Key below).

DESCRIPTION. Length ca $13 \mathrm{~mm}$, width of midbody pro- and metazonae 1.0 and $1.2 \mathrm{~mm}$, respectively (holotype); $\sigma^{7}$ paratype ca $11 \mathrm{~mm}$ long; + paratype ca $12 \mathrm{~mm}$ long, width of midbody pro- and metazonae 1.0 and $1.2 \mathrm{~mm}$, respectively. 


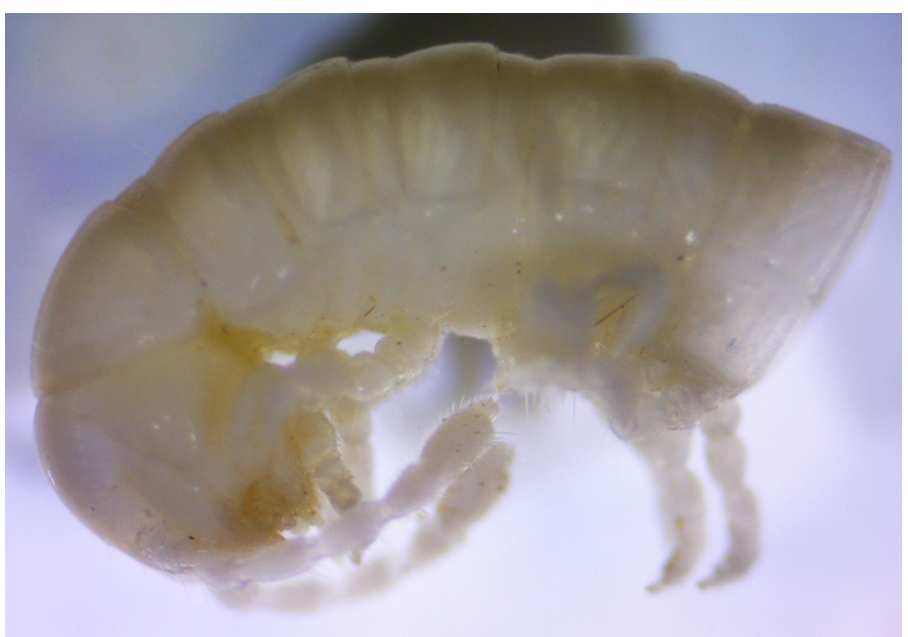

Fig. 29. Calloma pallidum sp.n., paratype female, head and anterior body segments, lateral view. Photo taken not to scale.

Рис. 29. Calloma pallidum sp.n., паратип самка, голова и передние сегменты тела, сбоку. Фотография сделана без масштаба.

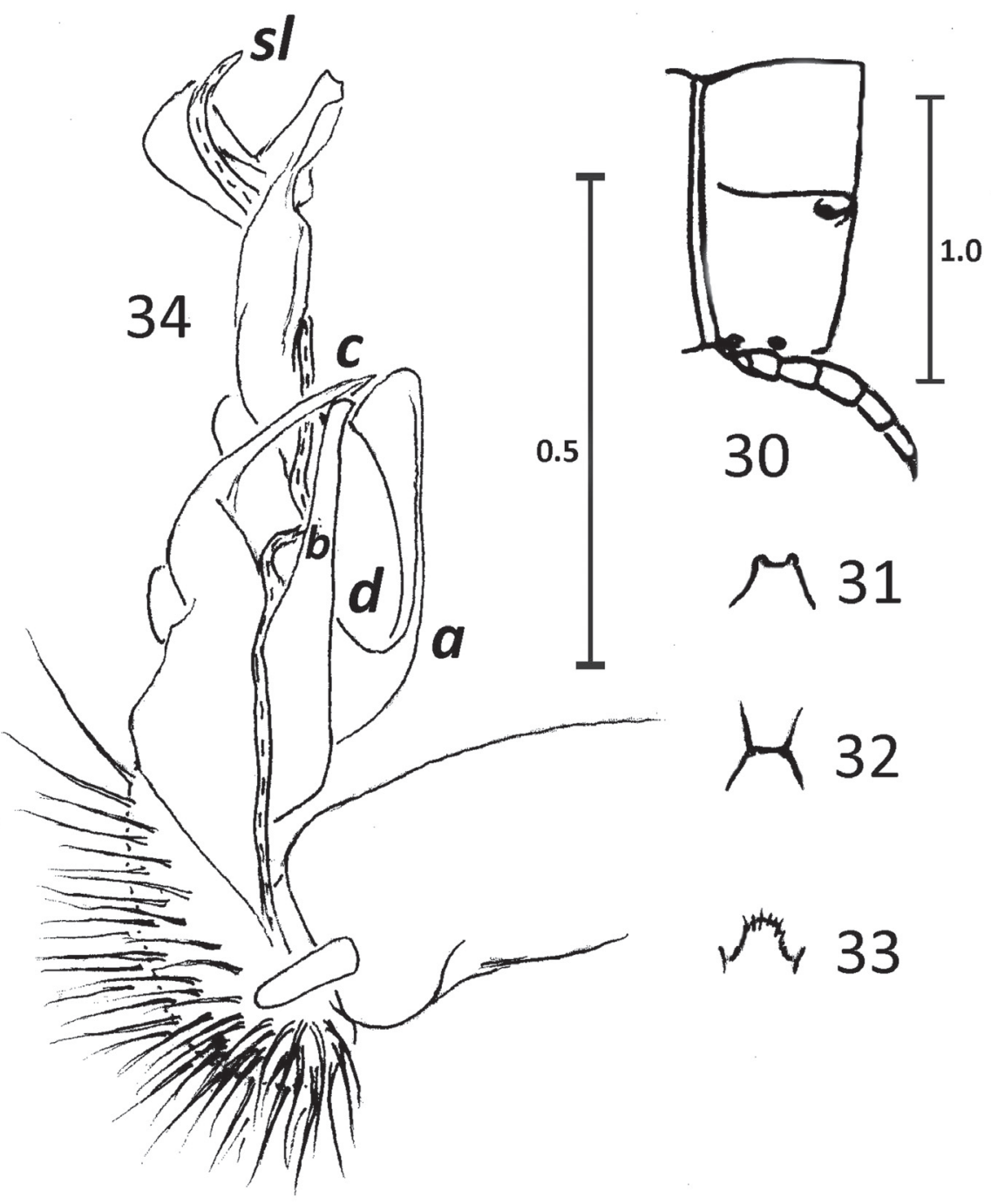

Figs 30-34. Calloma pallidum sp.n., holotype: 30 - midbody segment, lateral view; 31 - tip of epiproct, dorsal view; 32 hypoproct, ventral view; 33 - sternal lobe between coxae 4, subcaudal view; 34 - right gonopod, mesal view. Scale bars: 1.0 (30-33) and $0.5 \mathrm{~mm}(34)$

Рис. 30-34. Calloma pallidum sp.n., голотип,: 30 - среднетуловищный сегмент, сбоку; 31 - вершина эпипрокта, сверху; 32 гипопрокт, снизу; 33 - стернальная пластина между тазиками 4, почти сзади; 34 - правый гонопод, изнутри. Масштаб: 1,0 (3033) и 0,5 мм (34). 
Coloration entirely pallid, head slightly testaceous (Fig. 29).

In width, head $=$ collum $=$ segments $2-17$; body rapidly tapering towards telson thereafter. Clypeolabral region densely setose, vertex with $1+1$ setae. Antennae short, slightly clavate, reaching behind until midway of segment 2 when stretched dorsally; antennomere 6 highest and longest; antennomeres 2-5 subequal. Collum very broadly and regularly rounded, poorly rimmed. Tegument smooth and shiny throughout. Paraterga increasingly poorly developed, especially so in $q$, set low (at about midbody height) (Fig. 30); paraterga 2 and 3 best developed, ridge-like, but faint and bulge-shaped thereafter, delimited by vague sulci only dorsally, absent from segment $19^{\text {th }}$. Calluses absent. Tergal setae fully abraded, setation pattern untraceable. Ozopores close to caudal corner, located inside an ovoid groove, fully lateral. Axial line and transverse sulci wanting. Stricture faint, shallow and smooth. Pleurosternal carinae absent. Epiproct (Fig. 31) clearly emarginate at tip; subapical lateral papillae extremely small. Hypoproct (Fig. 32) subtrapeziform, setigerous papillae very small. Sterna poorly setose; cross-impressions faint, especially axial impressions; lobe between $\sigma^{\top}$ coxae 4 (Fig. 33) densely setose, linguiform. Legs short and stout, even in $\sigma^{7}$ about as long as midbody height; $\sigma^{7}$ tarsal brushes present until legs 7.

Gonopods complex (Fig. 34), coxae long and subcylindrical, bare; prefemoral part about as long as coxite and half as long as acropodite; femorite stout, with a dorsolateral lobe $(\boldsymbol{a})$, supporting a smaller lobe $(\boldsymbol{d})$ on ventral side; seminal groove running mesally before squeezing between two similar slender branches ( $b$ \& c); solenophore slender, slightly twisted, supporting a free solenomere by two apical lobes, solenomere slightly higher than solenophore.

\section{Caloma agametum Chamberlin, 1945}

MATERIAL. 1 incomplete $O^{7}$ (NMNHS), Papua New Guinea: Western Province, Finim Tel Plateau, rain forest, alt. $2300 \mathrm{~m}$, August 1975, B.S.E. (leg. P. Beron \& Ph. Chapman).

REMARKS. This species has already been recorded from the same area and even the same collection [Golovatch \& Stoev, 2009]. The very fine redescription by Hoffman [2005] requires no additional comments while the tribal position and distinguishing characters of Caloma have been discussed by Golovatch $\&$ Stoev [2009].

\section{Key to CALOMA SPECIES}

1. Coloration uniformly brown, paraterga present, produced acutely behind rear margin; transverse sulcus present, epiproct deeply emarginate; sternal lobe between $\sigma^{7}$ coxae 4 subtrapeziform; length $20 \mathrm{~mm}$, width $1.9 \mathrm{~mm}\left(\sigma^{7}\right)$; solenophore stout, ending in a subtriangular lobe
- Coloration different, paraterga poorly developed or absent; transverse metatergal sulci present or absent, epiproct deeply emarginate or truncate, sternal lobe between $\bigcirc^{7}$ coxae 4 different; length $\leq 15 \mathrm{~mm}$, width $\leq 1.2$ $\mathrm{mm}$; solenophore slender, trifid .................................. 2

2. Coloration reddish, transverse metatergal sulci present, paraterga mostly absent; epiproct truncate; sternal lobe between $\sigma^{7}$ coxae 4 subquadrate; length $15 \mathrm{~mm}$, width $0.9 \mathrm{~mm}\left(\mathrm{O}^{7}\right)$; gonopod femorite particularly stout, apical branches of solenophore slender, spiniform

C. insulare

- Coloration pallid, transverse metatergal sulci absent, paraterga very poorly developed (Fig. 30); epiproct deeply emarginate (Fig. 31); sternal lobe between $\sigma^{7}$ coxae 4 linguiform (Fig. 33); length 12-13 mm, width $1.2 \mathrm{~mm}$ $\left(\bigcirc^{7}\right)$; gonopod femorite less stout, two apical branches of solenophore lobe-shaped (Fig. 34)...

C. pallidum sp.n.

Tribe Aschistodesmini Jeekel, 1968

Genus Dendrogonopus Jeekel, 1964

Type species: Dendrogonopus robustus Jeekel, 1964, by original designation.

SPECIES INCLUDED: Dendrogonopus robustus Jeekel, 1964, from Beaufort River, Papua Province, Indonesia [Jeekel, 1964]; D. maculatus (Silvestri, 1895), from Moroka, Central Province, Bartholomew Mountains, Papua New Guinea [Silvestri, 1895; Jeekel, 1964]; and D. beroni sp.n., from Telefomin, Papua New Guinea.

\section{Dendrogonopus beroni sp.n.}

Figs 35-44.

HOLOTYPE $0^{\top}$ (NMNHS), Papua New Guinea: West Sepik Province, Telefomin, alt. 1700 m, 19 October 1975, leg. P. Beron.

NAME. Honours Peter Beron, a prominent Bulgarian zoologist and the collector of this species.

DIAGNOSIS. Differs from congeners by having a concave epiproct tip (Fig. 40), a subcircular sternal lobe between $\sigma^{7}$ coxae 4 (Fig. 42), and in gonopod process $\boldsymbol{p}$ being spiniform, process $\boldsymbol{k}$ tridentate and shorter than process $\boldsymbol{h}$, and the solenomere much shorter than both $\boldsymbol{k}$ and $\boldsymbol{h}$ (Figs 37, 38, 43 \& 44) (see also Key below).

DESCRIPTION. Length ca $30 \mathrm{~mm}$, width of midbody pro- and metazonae 3.0 and $4.0 \mathrm{~mm}$, respectively. Coloration generally brown (Figs 35 \& 36), but with a vivid pattern of whitish paraterga (caudal halves only) and a whitish, central, oblong axial spot in rear $1 / 2-2 / 3$ of metaterga. Legs, antennae and venter grey-brown, a little lighter than background.

In width, head $<$ collum $<$ segment $2<3-4<5-16$; body rapidly tapering towards telson thereafter. Clypeolabral region densely setose, vertigial region bare. Antennae rather long and slender, slightly clavate, reaching until midway of segment 3 when stretched dorsally; antennomeres 2 and 3 longest, only a little longer than subequal antennomers 4-6. Collum broadly rounded, slightly rimmed. Tegument smooth and shiny, but surface in pleurosternal region spiculate. Paraterga poorly developed (Figs 35, $36 \& 39$ ), set low (at about $1 / 3^{\text {rd }}$ of 

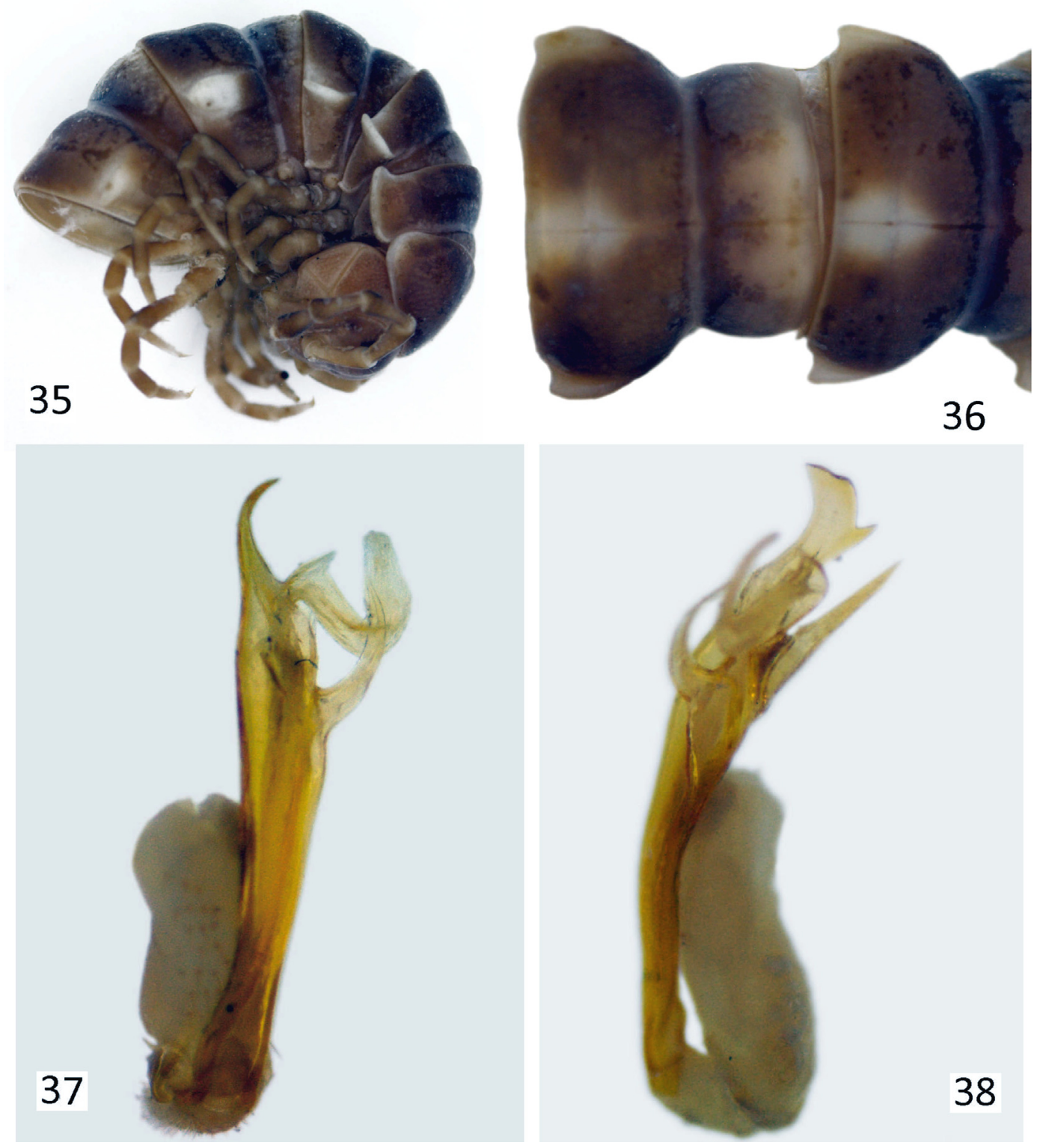

Figs 35-38. Dendrogonopus beroni sp.n., holotype: 35 - head and anterior segments, lateral view; 36 - midbody segments, dorsal view; 37 \& 38 - right gonopod, submesal and lateral views, respectively. Photos taken not to scale.

Рис. 35-38. Dendrogonopus beroni sp.n., голотип: 35 - голова и передние сегменты тела, сбоку; 36 - среднетуловищные сегменты, сверху; 37 и 38 - правый гонопод, соответственно почти изнутри и сбоку. Фотографии сделаны без масштаба.

body height); paraterga 2 broadly rounded both anteriorly and caudally, with a faint denticle near front corner, caudal corner a large rounded lobe produced behind rear tergal margin; paraterga 3 and 4 similarly thin and rounded, subhorizontal, slightly drawn behind the margin, but starting from paratergum 5 thick, flattened and not extending behind the margin. Calluses mostly thick, delimited by faint sulci both dorsally and ventrally. Tergal setae wanting, setation pattern untraceable. Ozopores located close to caudal corner, ventrolateral to lateral, superficial. Axial line thin, rather evident, visible both on pro- and metazonae. Transverse metatergal sulci superficial, not reaching bases of paraterga, visible on segments 5-18. Stricture between pro- and metazonae rather shallow, broad and smooth. Pleurosternal carinae small, complete, arcuated, low ridges traceable until segment 5 , thereafter wanting. Epiproct broad, rather poorly concave at tip; subapical lateral papillae extremely small (Fig. 40). Hypoproct semi-circular, setigerous papillae very small (Fig. 41). Sterna poorly setose, with rather deep cross-impressions and a small and a larger cone near each front and caudal coxa, respectively; lobe between coxae 4 nearly rounded, densely setose (Fig. 42). Legs rather long and slender, about 1.4-1.5 times as long as midbody height; tarsal brushes present until legs of segment 9 .

A pair of peculiar, very distinct, stump-shaped, densely setose, lateral protuberances behind gonopod 

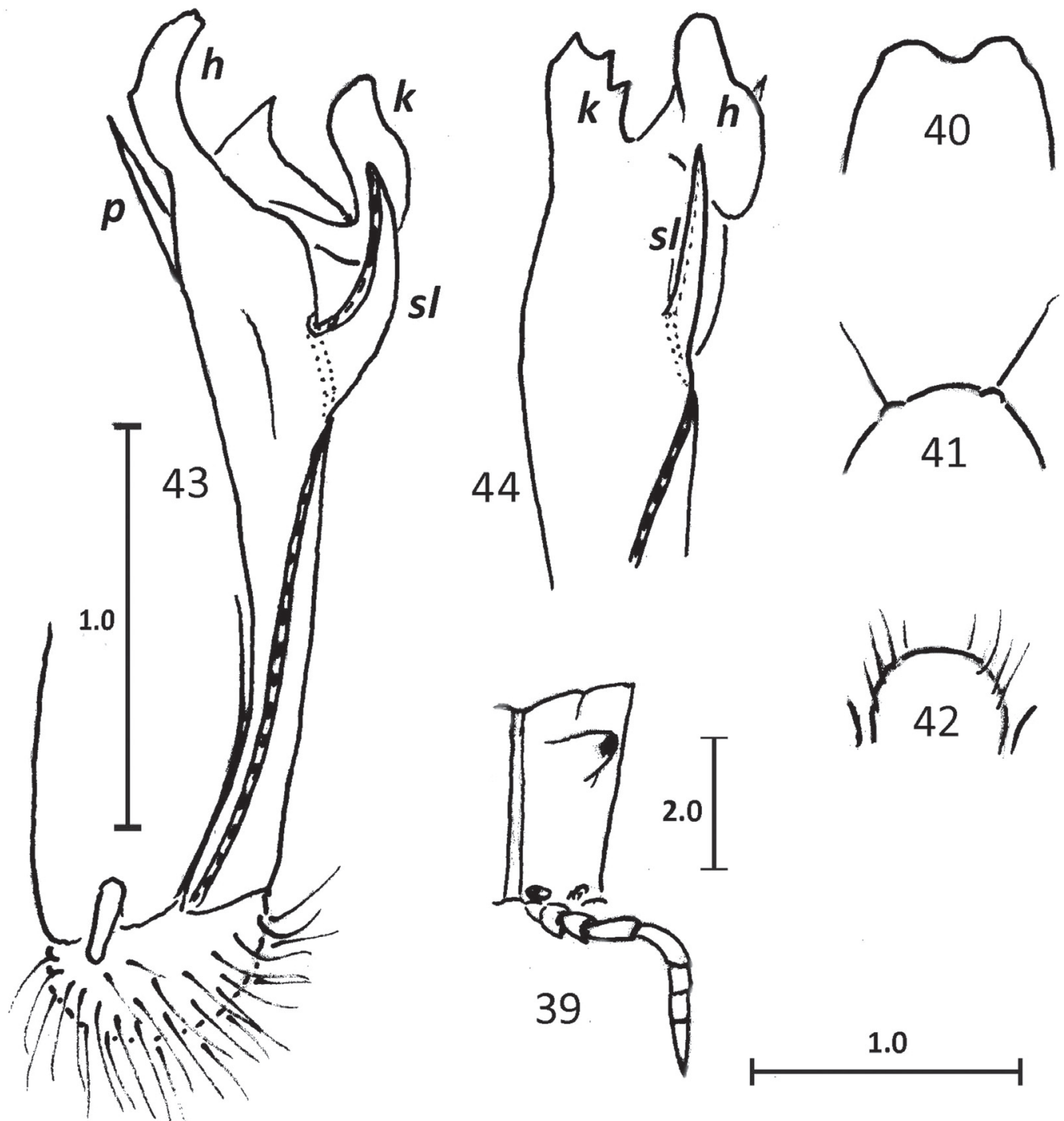

Figs 39-44. Dendrogonopus beroni sp.n., holotype: 39 - midbody segment, lateral view; 40 - tip of epiproct, dorsal view; 41 hypoproct, ventral view; 42 - sternal lobe between coxae 4, subcaudal view; $43 \& 44-$ left gonopod, mesal and ventral views, respectively. Scale bars: $2.0(39-42)$ and $1.0 \mathrm{~mm}(43 \& 44)$.

Рис. 39-44. Dendrogonopus beroni sp.n., голотип: 39 - среднетуловищный сегмент, сбоку; 40 - вершина эпипрокта, сверху; 41 - гипопрокт, снизу; 42 - стернальная пластина между тазиками 4 , почти сзади; 42 и 44 - левый гонопод, соответственно изнутри и снизу. Масштаб: 2,0 (39-42) и 1,0 мм (43 и 44).

aperture. Gonopods (Figs 37, 38, 43 \& 44) with very long, subcylindrical, nearly fully exposed coxae; telopodite fully exposed and closely appressed to coxite; prefemoral part conspicuously small, about $1 / 3^{\text {rd }}$ as long as acropodite; femorite very long and slender, clearly expanded towards apex; seminal groove running entirely on mesal side before entering onto a strong, subunciform solenomere $(\boldsymbol{s} l)$ on ventral side; apical part distal to $\boldsymbol{s} \boldsymbol{l}$ divided into a dorsal spine $(\boldsymbol{p})$ and two nearby lobes ( $\boldsymbol{h}$ and $\boldsymbol{k}$ ); lobe $\boldsymbol{k}$ tridentate, lobe $\boldsymbol{h}$ a little higher than $\boldsymbol{k}$.

\section{Key to DENDROGONOPUS SPECIES}

1.Epiproct truncate; sternal lobe between $\sigma^{7}$ coxae 4 moderately long, linguiform; gonopods: process $\boldsymbol{p}$ spiniform, process $\boldsymbol{k}$ lobe-shaped and about as high as a bifid narrow process $\boldsymbol{h}$, solenomere shorter than $\boldsymbol{k}$....

D. robustus

-Epiproct tip emarginated; sternal lobe between $\sigma^{7}$ coxae 4 and gonopods different

2. Epiproct tip deeply emarginate; sternal lobe between $\sigma^{7}$ coxae 4 subtrapeziform, tip truncated; gonopods: process $\boldsymbol{p}$ elongate subtriangular, process $\boldsymbol{k}$ subspiniform and shorter than a complex process $\boldsymbol{h}$, both about as high as solenomere...... D. maculatus

- Epiproct tip concave (Fig. 40); sternal lobe between $\sigma^{7}$ coxae 4 subcircular (Fig. 42); gonopods: process $p$ spiniform, process $\boldsymbol{k}$ tridentate and shorter than process $\boldsymbol{h}$, solenomere much shorter than both $\boldsymbol{k}$ and $\boldsymbol{h}$ (Figs 37, 38, $43 \& 44)$ D. beroni sp.n. 
ACKNOWLEDGEMENTS. We are most grateful to the Bulgarian-Russian Interacademician Exchange Programme which allowed the authors for brief research exchanges in 2013 to be made. Petar Beron (NMNHS) kindly provided certain important information concerning his 1975 trip to Papua New Guinea.

\section{References}

Attems C. 1914. Die indo-australischen Myriopoden // Archiv für Naturgeschichte. Bd.80A. S.1-398.

Chamberlin R.V. 1945. On some diplopods from the Indo-Australian Archipelago // American Museum Novitates. No.1282. P.1-43.

Golovatch S.I., Stoev P. 2009. New or poorly-known millipedes (Diplopoda) from Papua New Guinea, 1 // Arthropoda Selecta. Vol.18. Nos 3-4. P.125-130.

Golovatch S.I., Stoev P. 2010. New or poorly-known millipedes (Diplopoda) from Papua New Guinea, 3 // Arthropoda Selecta. Vol.19. No.3. P.145-152.

Golovatch S.I., Stoev P. 2011. Review of the millipede genus Eustrongylosoma Silvestri, 1896 in the island of New Guinea with descriptions of nine new species (Diplopoda, Polydesmida, Paradoxosomatidae) // International Journal of Myriapodology. Vol.5. P.1-26.

Golovatch S.I., Stoev P. 2014. Review of the Papuan millipede genus Silvattia Jeekel, 2009, with descriptions of three new species (Diplopoda: Polydesmida: Paradoxosomatidae: Eustrongylosomatini) // Telnov D. (Ed.): Biodiversity, Biogeo- graphy and Nature Conservation in Wallacea and New Guinea. Vol.2. P.145-152.

Helgen K.M., Leary T., Doria G., Amori G. 2008. Catalogue of Melanesian rodents in the Museum of Genova (Mammalia: Rodentia) // Annali del Museo Civico di Storia Naturale G. Doria. T.99. P.653-686.

Hoffman R.L. 1978. Diplopoda from Papuan Caves (Zoological Results of the British Speleological Expedition to Papua New Guinea, 1975, 4) // International Journal of Speleology. Vol.9 (for 1977). P.281-307.

Hoffman R.L. 2005. Zoological Results of the British Speleological Expedition to Papua New Guinea, 1975. A note on the characters and status of the genus Caloma Chamberlin, 1945 (Polydesmida: Paradoxosomatidae) // Myriapodologica. Vol.8. No.8. P.75-82.

Holthuis L.B. 1974. Notes on the localities, habitats, biology, colour and vernacular names of New Guinea freshwater crabs (Crustacea, Decapoda, Sundathelpusidae) // Zoologische Verhandelingen. Deel 13. P.3-47.

Jeekel C.A.W. 1964. Notes on the genus Akamptogonus Attems, with descriptions of a new genus and species from New Guinea (Diplopoda, Polydesmida) // Nova Guinea, Zoology. Vol.29. P.105-113.

Jeekel C.A.W. 1968. On the classification and geographical distribution of the family Paradoxosomatidae (Diplopoda, Polydesmida) // Academisch Proefschrift (PhD Thesis), Rotterdam. 162 pp.

Silvestri F. 1895 Chilopodi e diplopodi della Papuasia // Annali di Museo Civico di Storia Naturale di Genova, Serie 2. T.14(34). P.441-450.

Responsible editor K.G. Mikhailov 